\title{
THE LINEAR DIFFERENCE EQUATION OF THE FIRST ORDER
}

\author{
By E. W. BARNES. \\ [Received September 16th, 1904-Read November 10th, 1904.]
}

\section{CONTENTS.}

1. Introduction. Historical Résuné.-2. Reduction of the General Difference Equation $\phi(z) f^{\prime}(z+\omega)-\chi(z) f(z)=\psi(z)$ to Two Standard Equations.-3. Definitions.-4-7. Solution of $f(z+\omega)=\phi(z) f(z)$, where $\phi(z)$ is holomorphic, of tinite order, without extraneous exponential factor, with zeros all negative with respect to $\omega .-8$. Solution of $f(z+\omega)=\phi(z) f(z)$, where $\phi(z)$ is holomorphic, of finite order, without extraneous exponential factor, the zeros being positive with respect to $\omega .-9,10$. Solution of $f(z+\omega)=\mu(z) f(z)$, where $\mu(z)$ is meromorphic and possibly of infinite order.-11, 12. The Riemann $\zeta$ Function and its Modification. Solution of $f(z+\omega)-f(z)=G(z)$, where $G(z)$ is an integral function. -13 . Completion of $\$ 10 .-14,15$. Other Forms for the Solution of $f(z+\omega)=\mu(z) f(z)$. -16. Extension to Cases where $\mu(z)$ has Repeated Zeros or Poles or is of Multiple Sequence.-17-22. Solution of $f(z+\omega)-f(z)=\mu(z)$ in Three Different Forms. - 23. The Nature of the Solution of $\phi(z) f(z+\omega)-\chi(z) f^{\prime}(z)=\psi(z)$. 24. Examples. Conclusion.

1. In the present paper it is proposed to investigate the nature of the functions defined by linear difference equations of the first order. The three main questions to be considered are (i.) the existence of a solution, (ii.) its analytical expression, (iii.) its place among transcendental functions. These questions are, of course, bound up with one another: the first is obviously contained in the second; and the third has already formed the subject of a separate paper, ${ }^{*}$ in which the general results here obtained were assumed.

Linear difference equations arose historically in arithmetical investigations connected with the theory of interpolation and with the necessarily discontinuous nature of physical experiments. And, in consequence, until recently such equations have been considered only in cases where the variable is a real number. References to many investigations of this nature are given by Boole. $t$ The first investigation where the variable was supposed to assume all complex values appears to have been given by Guichard. $\ddagger$ By means of Hermite's coupures he proves that there exists a holomorphic solution of

$$
f(x+1)-f(x)=\mu(x)
$$

* Proc. London Math. Soc., Ser. 2, Vol. 2, pp. 280-292.

† Boole, Finite Differences, Third Edition, pp. 232, 263.

I Guichard, Annales de l'École Nor'nnale Supúrieu'e (1887), Sér. 3, T. 1v., pp. 361-380. 
when $\mu(x)$ is holomorphic. He further gives a criterion for the nature of the holomorphic function $\nu(x)$ that

$$
f(x+1)-\nu(x) f(x)=0
$$

may admit a holomorphic solution.

Appell* was the next to give an expression for the holomorphic solution of

$$
f(x+1)-f(x)=\mu(x)
$$

when $\mu(x)$ is holomorphic.

Then Mellin, $t$ starting from a result in the theory of gamma functions due to Prym, $\ddagger$ considered the nature of the solution of

$$
f(x+1)-r(x) f(x)=s(x)
$$

where $r(x)$ and $s(x)$ are rational functions of $x$.

More recently there has appeared a paper by Hurwitz. $\$$ Some of his results anticipate those of the present investigation; they are indicated in the text. Hurwitz solved independently, but by substantially the same method, the problem considered by Appell, and showed how to solve the equation

$$
\phi(x) f(x+1)-\chi(x) f(x)=\psi(x)
$$

where $\phi(x), \chi(x)$, and $\psi(x)$ are meromorphic functions.

2. The linear difference equation of the first order may be written

$$
\phi(z) f(z+\omega)-\chi(z) f(z)=\psi(z),
$$

where we assume $\phi(z), \chi(z)$, and $\psi(z)$ to be analytic functions of $z$. It is at once evident that we may reduce this equation to two others of simpler type,

and

$$
\frac{f_{1}(z+\omega)}{f_{1}(z)}=\frac{\phi(z)}{\chi^{(z)}}
$$

and that then

$$
f_{2}(z+\omega)-f_{2}(z)=\frac{\psi(z)}{\chi(z)} f_{1}(z),
$$

$$
f^{\prime}(z)=\frac{f_{2}(z)}{f_{1}(z)} .
$$

For, substituting $f_{1}(z) f(z)$ for $f_{2}(z)$ in the second equation, we have

$$
f_{1}(z+\omega) f(z+\omega)-f_{1}(z) f^{\prime}(z)=\frac{\psi(z)}{\chi(z)} f_{1}(z) ;
$$

* Appell, Lionville (1891), Sér. 4, T. vIr., pp. 157-219, especially chapter i.

+ Mellin, Acta Mathematica, T. xv., pp. 317-384.

$\ddagger$ Prym, Crelle, Bd. Lxxxir., pp. 165-172.

Hurwitz, Acta Mathematica (1897), T. xx., pp. 285-312; T. xxı., p. 243 . 
so that, by the first equation,

$$
\phi(z) f(z+\omega)-\chi(z) f(z)=\psi(z),
$$

which is the equation from which we started.

We may then regard (A) and (B) as the two fundamental equations. Although, by taking logarithms, we may reduce these to a common form, yet it is convenient to consider each separately. By so doing, we not only avoid the deviation from uniformity introduced by the logarithm, but we obtain two expressions representing solutions of either equation which correspond in some degree to the expression by the theorems of Weierstrass and Mittag-Leffler of a uniform transcendental function.

3. We propose to limit ourselves to the case when the coefficients in the difference equation are uniform functions with a single essential singularity at infinity. It is obvious that, with such a restriction, we may take $\phi(z), \chi(z)$, and $\psi(z)$ to be integral functions (holomorphic functionsfonctions entières).

In three papers* recently published I have analysed integral functions and introduced certain definitions which it is convenient to repeat here.

A simple integral function is a function which may be expressed as a single Weierstrassian product, whose $n$-th zero $a_{n}$ depends solely upon $n$ and certain definite constants, and which is such that the law of dependence of $a_{n}$ upon $n$ is the same for all but a finite number of zeros. Tha function is called a non-repeated function if the $n$-th primary factor of Weierstrass's product does not correspond to a zero of order depending upon $n$. If there is such dependence, it is called a repeated simple integral function.

Functions of multiple linear sequence are functions whose general zero is of the type

$$
f\left(a+m_{1} \omega_{1}+\ldots+m_{r} \omega_{r}\right),
$$

the $m$ 's being the integers which define the particular zero.

The order of a simple non-repeated integral function is a real positive quantity $\rho$ such that $\sum_{n=1}^{\infty} \frac{1}{\left|a_{n}\right|^{p+\epsilon}}$ converges and $\sum_{n=1}^{\infty} \frac{1}{\left|a_{n}\right|^{\rho-\epsilon}}$ diverges, however small the real positive quantity $\epsilon$ may be. When $\rho$ is dependent upon $n$ the function is of infinite order.

Analogous definitions can be given for the order of repeated functions

"(1) "A Memoir on Integral Functions," Phil. Tians. Roy. Soc. (A), Vol. 199, pp. 411-500; (2) "The Classification of Integral Functions," Camb. Phil. Trans., Vol. xax., pp. 322-355; (3) "The Asymptotic Expansion of Integral Functions of Multiple Linear Sequence," ibid., Vol. xix., pp. 426-439. 
and functions of multiple linear sequence. For them I may conveniently refer to the papers cited.

Suppose now that we have the equation (A),

$$
\frac{f_{1}(z+\omega)}{f_{1}(z)}=\frac{\phi(z)}{\chi^{(z)}}
$$

where $\phi(z)$ and $\chi(z)$ are simple non-repeated integral functions of finite or infiuite order. The equation may be written

$$
\frac{f_{1}(z+\omega)}{f_{1}(z)}=e^{G(z)} \frac{\prod_{n=1}^{\infty}\left[\left(1-\frac{z}{a_{n}}\right) e^{f_{11}(z)}\right]}{\prod_{n=1}^{\infty}\left[\left(1-\frac{z}{b_{n}}\right) e^{g_{n}(z)}\right]}
$$

where $G(z)$ is an integral function.

Now it is evident that a solution of

$$
\frac{f_{1}(z+\omega)}{f_{1}(z)}=e^{G(z)}
$$

is $e^{G_{1}(z)}$ where $G_{1}(z)$ is a solution of $f(z+\omega)-f(z)=G(z)$, which is of the for'm of equation $(B)$.

We limit ourselves then to the case when the quotient $\frac{\phi(z)}{\chi(z)}$, expressed as a quotient of two Weierstrassian products, involves no extraneous exponential factor.

4. In the first place, we consider the equation

$$
\frac{f(z+\omega)}{f(z)}=\phi(z)
$$

where $\phi(z)$ is a holomorphic function of finite order with no extraneous exponential factor whose zeros are all negative with respect to $\omega$. By this we mean that, if we draw a line from the origin to the point $\omega$, the zeros all lie in the half plane which is on the other side from $\omega$ of the perpendicular through the origin to $O \omega$. This part of the plane is shaded in the figure (p. 442).

When expressed as a Weierstrassian product

$$
\phi(z)=\prod_{n=1}^{\infty}\left[\left(1+\frac{z}{a_{n}}\right) e^{\sum_{s=1}^{\rho-1} \frac{(-)^{*}}{s}\left(\frac{z}{a_{n}}\right)}\right]
$$

where $\rho$ is the integer next greater than the order of the function, and 


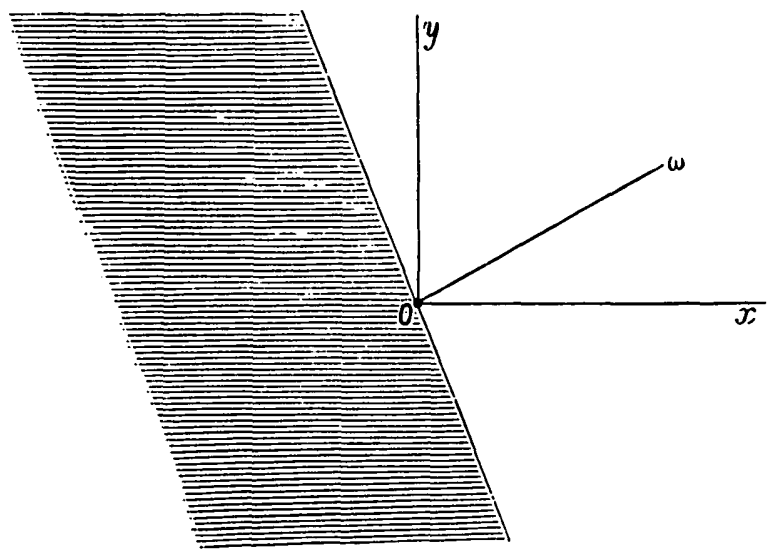

$\frac{\alpha_{1}}{\omega}, \ldots, \frac{\alpha_{n}}{\omega}, \ldots$ form a series of quantities whose real parts are positive and whose moduli are arranged in non-decreasing order of magnitude.

Take the simple modified gamma function*

$$
\Gamma_{1}^{-1}(z)=\omega^{-: \omega} e^{\gamma: \omega} z \prod_{m=1}^{\alpha}\left[\left(1+\frac{z}{m \omega}\right) e^{-z: m \omega}\right],
$$

which satisfies the difference equation

$$
\frac{f(z+\omega)}{f(z)}=z .
$$

As usual, we write

$$
\psi_{1}^{(r)}(z)=\frac{d^{r}}{d z^{r}} \log \Gamma_{1}(z) \quad(r=1,2, \ldots)
$$

Construct the product

$$
P(z)=\prod_{n=1}\left[\frac{\Gamma_{1}\left(z+a_{n}\right)}{\Gamma_{1}\left(a_{n}\right)} e^{-\sum_{s=1}^{o-1} \frac{z^{x}}{s !} \psi_{1}^{(s)}\left(a_{n}\right)}\right]
$$

where $\sigma$ is an integer to be presently determined.

The first $k-1$ terms of this product for $P(z)$ are evidently convergent so long as $P(z)$ is finite. If we suppose that $|z|<\left|a_{k}\right|$, we may expand $\log \frac{\Gamma_{1}\left(z+a_{n}\right)}{\Gamma_{1}\left(a_{n}\right)}$ by Taylor's theorem and write the remaining terms in the product for $P(z)$ in the form

$$
\exp \left[\sum_{n=k}^{\infty} \sum_{s=\sigma}^{\infty} \psi_{1}^{(s)}\left(\alpha_{n}\right) \frac{z^{s}}{s !}\right]
$$

* This function is chosen with form given on account of its analogy with multiple gamma funotions. Its theory was worked out by the author in the Mcssenger of Mathematics, Vol. xxix., pp. 64-12s. 
Now

$$
\psi_{1}^{(1)}(z)=-\frac{\gamma}{\omega}+\frac{\log \omega}{\omega}-\frac{1}{z}-\sum_{n=1}^{\infty}\left\{\frac{1}{z+m \omega}-\frac{1}{m \omega}\right\},
$$

and therefore, if $s>1$,

$$
\psi_{1}^{(s)}(z)=(-)^{s}(s-1) ! \sum_{m=0}^{\infty} \frac{1}{(z+m \omega)^{s}} .
$$

The remaining terms in the product for $P(z)$ may therefore be written (assuming that $\sigma>1$ )

$$
\exp \left[\sum_{n=k}^{\infty} \sum_{s=\sigma}^{\infty} \sum_{m=0}^{\infty} \frac{(-z)^{s}}{s\left(\alpha_{n}+m \omega\right)^{s}}\right]=\exp Z, \text { say. }
$$

Consider first the double series

$$
\sum_{s=\sigma}^{\infty} \sum_{m=0}^{\infty} \frac{(-z)^{s}}{s\left(a_{n}+m \omega\right)^{s}}
$$

Fach series $\sum_{m=0}^{\infty} \frac{(-z)^{s}}{s\left(\alpha_{n}+m \omega\right)^{s}}$, being equal to $\psi_{1}^{(s)}\left(a_{n}\right) \frac{z^{s}}{s !}$, is absolutely convergent for all values of $s$.

Again, since $-a_{n}$ is negative with respect to $\omega$, we may use the asymptotic expansion for $\psi_{1}^{(s)}\left(\alpha_{n}\right)$, and we see that

$$
\sum_{s=\sigma}^{\infty} \sum_{m=0}^{\infty} \frac{(-z)^{s}}{s\left(\alpha_{n}+m \omega\right)^{s}} \quad \text { converges with } \sum_{s=\sigma}^{\infty} \frac{z^{s}}{s !} \frac{(-)^{s}(s-2) !}{a_{n}^{s-1}},
$$

and is thus absolutely convergent if $\left|z / \alpha_{n}\right|<1$.

For when $|z|$ is large and not near the line of poles of $\psi_{1}^{(1)}(z)$ we have asymptotically*

$$
\psi_{1}^{(1)}(z)=\frac{1}{\omega} \log z+\left(\frac{z}{\omega}-\frac{1}{2}\right) \frac{1}{z}+\sum_{s=1}^{\infty} \frac{(-)^{r}}{z^{r+1}} B_{r+1}(\omega)
$$

and $\quad \psi_{1}^{(s)}(z)=\frac{(-)^{s}(s-2) !}{\omega z^{s-1}}+$ smaller terms.

Hence, by a result due to Cauchy, $t$ the double series is equal to

We may therefore write

$$
\sum_{m=0}^{\infty} \sum_{s=\sigma}^{\infty} \frac{(-z)^{s}}{s\left(a_{n}+m \omega\right)^{s}} .
$$

$$
Z=\sum_{n=k}^{\infty} \sum_{m=0}^{\infty} \sum_{s=\sigma}^{\infty} \frac{(-z)^{s}}{s\left(\alpha_{n}+m \omega\right)^{s}}
$$

- Loc. cit., Part Iv.

$\dagger$ Analyse Algébrique, Note vir. ; Résumés Analytiques, $\$ 8$. 
and therefore

$$
\begin{aligned}
|Z| & <\frac{1}{\sigma} \sum_{n=k}^{\infty} \sum_{m=0}^{\infty} \sum_{s=\sigma}^{\infty} \frac{|z|^{s}}{a_{n}+\left.m \omega\right|^{s}} \\
& <\frac{1}{\sigma} \sum_{n=k}^{\infty} \sum_{m=0}^{\infty} \frac{|z|^{\sigma}}{a_{n}+\left.m \omega\right|^{\sigma}} /\left\{1-\left|\frac{z}{a_{n}+m \omega}\right|\right\},
\end{aligned}
$$

provided $\left|z /\left(a_{n}+m \omega\right)\right|<1$, that is to say, remembering the distribution of the points $a_{n}$, provided $|z|<u_{k}$.

If now $\mu$ be the minimum value of $1-\left|z /\left(a_{n}+m \omega\right)\right|$, we have

$$
|Z|<\frac{|z|^{\sigma}}{\mu^{\sigma}} \sum_{n=k}^{\infty} \sum_{m=0}^{\infty} \frac{1}{\left|\alpha_{n}+m \omega\right|^{\sigma}} .
$$

It is necessary now to investigate series of this type.

5. We proceed to prove that, if

$$
a_{1}, \alpha_{2}, \ldots, a_{n}, \ldots, \quad \beta_{1}, \beta_{2}, \ldots, \beta_{n}, \ldots
$$

form two series of quantities whose graphic representations lie within a quadrant of the Argand diagram, and whose moduli form in each case a non-decreasing series, and if $\sum_{n=1}^{\infty} \frac{1}{\left|\alpha_{n}\right|^{\rho}}$ and $\sum_{n=1}^{\infty} \frac{1}{\left|\beta_{n}\right|^{\sigma}}$ are absolutely convergent, then is

$$
\sum_{n=1}^{\infty} \sum_{m=1}^{\infty} \frac{1}{\left(a_{n}+\beta_{m}\right)^{s}}
$$

an absolutely convergent series, provided $s$ is equal to or greater than the greater of the two quantities $2 \rho$ and $2 \sigma$.

Since the quantities $\alpha_{n}$ and $\beta_{n}$ lie within a quadrant of the Argand diagram, we readily see from a figure that

$$
\left|a_{n}+\beta_{m}\right|^{2}>a_{n}^{2}+b_{m}^{2}
$$

where

$$
a_{n}=\left|a_{n}\right| \text { and } b_{m}=\left|\beta_{n n}\right| \text {. }
$$

In the limiting case when the quantities lie respectively on the arms of the quadrant this inequality becomes an equality.

The modulus of the series to be investigated is therefore

$$
\gtrless \sum_{n=1}^{\infty} \sum_{m=1}^{\infty} \frac{1}{\left(a_{n}^{2}+b_{n n}^{2}\right)^{\frac{1}{s^{3}}}} \text {. }
$$

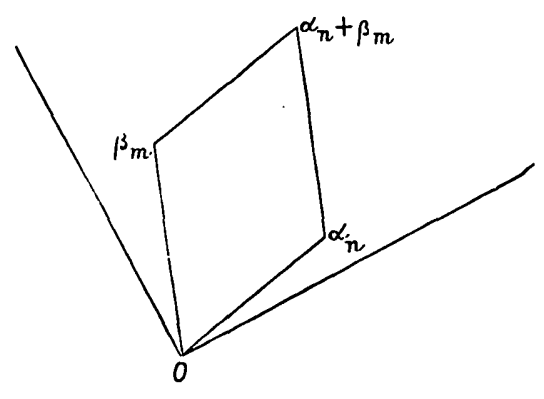


Now $a_{n}^{2}+b_{m}^{2}>2 a_{n} b_{m}$; hence the modulus is

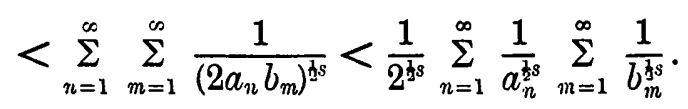

Thus the series is absolutely convergent, in any of the four ways in which we may sum it, and converges to the same definite limit provided $s$ is greater than or equal to the greater of the two quantities $2 \rho$ and $2 \sigma$. The proposition is therefore established.

6. Return now to the series $\sum_{n=k}^{\infty} \sum_{m=0}^{\infty} \frac{1}{\left(a_{n}+m \omega\right)^{\sigma}}$, which arose in $\$ 4$. The only restriction which we have so far imposed upon $\sigma$ is that it is greater than 1. The quantities $a_{1}, \ldots, a_{n}, \ldots$ were taken to be positive with respect to the $\omega$ 's', which is equivalent to saying that the $a$ 's lie in the Argand diagram within an angle of $90^{\circ}$ on either side of the positive direction of the axis of $\omega$.

We assumed that $\sum_{n=1}^{\infty} \frac{1}{\left|a_{n}\right|^{p}}$ is convergent, and we know that $\sum_{m=1}^{\infty} \frac{1}{|m \omega|^{1+e}}$ is convergent, if $\epsilon>0$.

Hence the series $\sum_{n=k}^{\infty} \sum_{m=0}^{\infty} \frac{1}{\left(a_{n}+m \omega\right)^{\sigma}}$ is absolutely convergent, provided $\sigma>2 \rho$ and $\sigma>2$.

With these limitations on $\sigma$ we see that $P(z)$ is absolutely convergent at all points of the plane except the poles of the functions $\Gamma_{1}\left(z+a_{n}\right)$. $P(z)$ is therefore a one-valued meromorphic function of $z$ with these poles and no zeros.

7. Consider now the quotient $P(z+\omega) / P(z)$.

Since $\Gamma_{1}(z+\omega)=z \Gamma_{1}(z)$, we evidently have

$$
\begin{aligned}
\frac{P(z+\omega)}{P(z)} & =\prod_{n=1}^{\infty}\left[\left(z+\alpha_{n}\right) e^{-\sum_{s=1}^{o-1} \frac{\psi_{1}^{(s)}\left(\alpha_{n}\right)}{s !}\left[(z+\omega)^{s}-z^{s}\right]}\right] \\
& =\prod_{n=1}^{\infty}\left[\left(1+\frac{z}{\alpha_{n}}\right) e^{\left.\sum_{s=1}^{\rho-1} \frac{(-)^{s}}{s}\left(\frac{z}{a_{n}}\right)^{s}\right]}\right. \\
& \times \prod_{n=1}^{\infty}\left[\exp \left\{\sum_{s=1}^{\rho-1} \frac{(-)^{s-1}}{s}\left(\frac{z}{a_{n}}\right)^{s}-\sum_{s=1}^{\sigma-1} \frac{\psi_{1}^{(s)}\left(a_{n}\right)}{s !}\left[(z+\omega)^{s}-z^{s}\right]\right\}\right] .
\end{aligned}
$$

Now $P(z+\omega), P(z)$ and the first product on the right-hand side are each absolutely convergent. The second product on the right-hand side is 
therefore absolutely convergent and may be written

$$
\exp \left\{-\sum_{m=0}^{\sigma-2} c_{m} z^{m}\right\}
$$

where the $c$ 's are definite finite functions of the $a$ 's. ${ }^{*}$

We have now

$$
\frac{P(z+\omega)}{P(z)}=\phi(z) \exp \left\{-\sum_{m=0}^{\sigma-2} c_{m} z^{m}\right\}
$$

Hence the function $P(z) \exp \left[\sum_{m=0}^{\sigma-2} c_{m} S_{m}(z)\right]$, where $S_{m}(z)$ is the Bernoullian function of $z, \dagger$ satisfies the difference equation with which we started in $\S 4$,

$$
f(z+\omega)-\phi(z) f(z)=0 .
$$

The general solution of this difference equation is the particular solution multiplied by a simply periodic function of $z$ of period $\omega$.

The principal solution is a meromorphic function with no zeros, all of whose poles are at the points

$$
z=-\alpha_{n}-m \omega \quad(m=0,1, \ldots, \infty),
$$

the zeros of $\phi(z)$ being at the points $z=-a_{n}$.

We propose to say that the poles of the particular solution are at the points negatively congruent to the zeros of $\phi(z)$. These points are the doubly infinite series formed by sequences of points stretching out negatively at intervals $\omega$ from the points $z=-a_{n}$ as in the diagram.

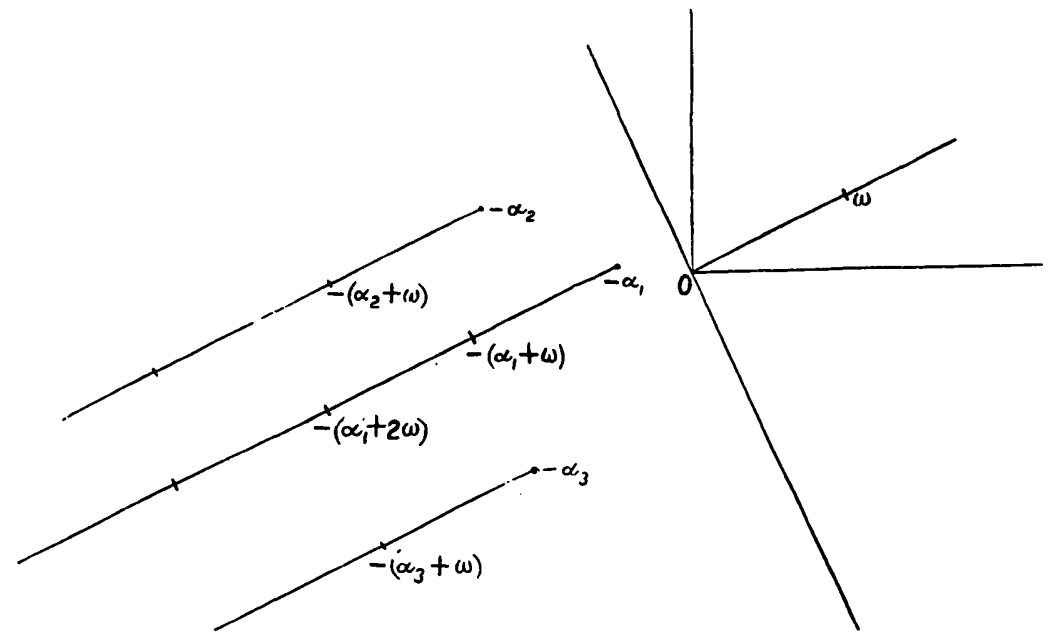

* In certain special cases I have evaluated the c's by means of asymptotic expansions. See "The Theory of the Double Gamma Function," Phil. Trans. Roy. Soc. (A), Vol. 146, pp. 265-387, $\$ \$ 40$ and 41 ; and, for the extension to multiple gamma functions, Camb. Phil. Trans., Vol. xIX., pp. 374-425, Part 3.

+ Vide Messenger of Mathenatics, Vol. xxix., pp. 64-128, Part 2. 
The reciprocal of the particular solution is a non-repeated integral function of double sequence.

In the course of the proof we have imposed on $\phi(z)$ the limitation that all its zeros should be negative with respect to $\omega$. But it is evident that, in general, a finite number may be at finite points on the positive side of or actually on the perpendicular through the origin to $O \omega$. For, corresponding to such points, we have in general a finite primary gamma factor multiplying the solution obtrined. The exceptional case, which may be readily treated, occurs when $-\left(\alpha_{n}+m \omega\right)$ is, for some values of $m$ and $n$, identically zero.

8. We will consider next the solution of the difference equation

$$
f(z+\omega)-\phi(z) f(z)=0,
$$

where $\phi(z)$ is a simple non-repeated integral function of finite order without extraneous exponential factor, and all but a finite number of its finite zeros lie on the positive side of the perpendicular through the origin to $O \omega$.

Neglecting the exceptional zeros which may be treated separately, we may put

$$
\phi(z)=\prod_{n=1}^{\infty}\left[\left(1-\frac{z}{\beta_{n}}\right) e^{\rho+\infty=1} \frac{1}{s}\left(\begin{array}{c}
z \\
\beta_{n}
\end{array}\right)^{s}\right]
$$

where $\rho$ is the integer next greater than the order of the function and $\beta_{1} / \omega, \ldots, \beta_{n} / \omega, \ldots$ form a series of quantities which have their real parts positive, and are arranged so that their moduli are in non-decreasing order of magnitude. We construct the product

$$
P(z)=\prod_{n=1}^{\infty}\left[\frac{\Gamma_{1}\left(\omega+\beta_{n}\right)}{\Gamma_{1}\left(\omega+\beta_{n}-z\right)} \exp \left\{\sum_{s=1}^{\sigma-1} \frac{(-z)^{s}}{s !} \psi_{1}^{(s)}\left(\omega+\beta_{n}\right)\right\}\right] .
$$

The terms of $P(z)$ after the first $(k-1)$ are equal to

$$
\exp \left[\sum_{n=k}^{\infty} \sum_{s=\sigma}^{\infty}\left\{-\frac{(-z)^{s}}{s !} \psi_{1}^{(s)}\left(\omega+\beta_{n}\right)\right\}\right]
$$

provided

$$
|z|<\omega+\beta_{k} \text {. }
$$

Therefore, if $\sigma>1$, we see, as in $\$ 4$, that they may be written

$$
\exp \left[-\sum_{n=l}^{\infty} \sum_{s=\sigma}^{\infty} \sum_{n=0}^{\infty} \frac{z^{s}}{s\left(\omega+\beta_{n}+m \omega\right)^{s}}\right]=\exp \left[-\sum_{n=k}^{\infty} \sum_{s=\sigma}^{\infty} \sum_{n=1}^{\infty} \frac{z^{s}}{s\left(\beta_{n}+m \omega\right)^{s}}\right] .
$$


Exactly as in $\$ 6$, we can now demonstrate that the product $P(z)$ is absolutely convergent, provided $\sigma>2 \rho$ and $\sigma>2$. Now

$$
\begin{aligned}
\frac{P(z+\omega)}{P(z)}= & \prod_{n=1}^{\infty}\left[\frac{\Gamma_{1}\left(\omega+\beta_{n}-z\right)}{\Gamma_{1}\left(\beta_{n}-z\right)} \exp \sum_{s=1}^{\sigma-1} \psi_{1}^{(s)}\left(\omega+\beta_{n}\right) \frac{(-)^{s}}{s !}\left\{(z+\omega)^{s}-z^{s}\right\}\right] \\
= & \phi(z) \prod_{n=1}^{\infty} \exp \left[\log \beta_{n}-\sum_{s=1}^{p-1} \frac{1}{s}\left(\frac{z}{\beta_{n}}\right)^{s}\right. \\
& \left.\quad+\sum_{s=1}^{\sigma-1} \frac{(-)^{s}}{s !} \psi_{1}^{(s)}\left(\omega+\beta_{n}\right)\left\{(z+\omega)^{s}-z^{s}\right\}\right] \\
= & \phi(z) \exp \left\{-\sum_{s=0}^{\sigma-2} d_{s} z^{s}\right\},
\end{aligned}
$$

where the quantities $d$ are finite functions of the $\beta$ 's. Thus a solution of

is

$$
\begin{aligned}
& f(z+\omega)-\phi(z) f(z)=0 \\
& P(z) \exp \left\{\sum_{s=0}^{\sigma-2} d_{s} S_{s}(z)\right\},
\end{aligned}
$$

and the general solution consists of this function multiplied by an arbitrary simply periodic function of $z$ of period $\omega$. The particular solution thus obtained is an integral function of $z$ whose zeros are at the points positively congruent to the zeros of $\phi(z)$, these zeros excluded.

When $\omega=1$ our restriction is that all but a finite number of zeros of $\phi(z)$ should lie to the right of the imaginary axis, in order that a particular solution of $f(z+1)=\phi(z) f(z)$, where $\phi(z)$ is holomorphic, should itself be holomorphic. It may be compared with Guichard's result.*

9. It is now evident that there exists a meromorphic solution of

$$
f(z+\omega)-\mu(z) f(z)=0
$$

where $\mu(z)$ is a meromorphic function of $z$ which is a quotient of two simple non-repeated integral functions of finite order which when expressed as Weierstrassian products involve no extraneous exponential factor.

Under the specified conditions we may write

$$
\mu(z)=\prod_{n=1}^{\infty}\left\{\frac{\left[\left(1+\frac{z}{\alpha_{n}}\right) e^{\sum_{s=1}^{\rho-1} \frac{(-z)^{s}}{s a_{n}^{s}}}\right]\left[\left(1-\frac{z}{\beta_{n}}\right) e^{\stackrel{\sum_{s=1}^{-1}}{s} \frac{1}{s}\left(\frac{z}{\beta_{n}}\right)^{\prime}}\right]}{\left[\left(1+\frac{z}{\gamma_{n}}\right) e^{\left.\sum_{s=1}^{s-1} \frac{(-z)^{s}}{s \gamma_{n}^{s}}\right]}\right]\left[\left(1-\frac{z}{\delta_{n}}\right) e^{\left.\sum_{s=1}^{s-1} \frac{1}{s}\left(\frac{z}{\delta_{n}}\right)^{\prime}\right]}\right\}}\right\}
$$

* Loc. cil., §1, p. 376. See also Hurwitz, loc. cit., p. 312. 
where $\rho, \sigma, \tau, v$ are integers next greater than the orders of the respective products, and $a_{1} / \omega, \ldots, a_{n} / \omega, \ldots$, and the three corresponding sequences form series whose real parts are positive and whose moduli are arranged in non-decreasing order of magnitude.

And now, from the results of $\S \S 7$ and 8 , a solution of

$$
\begin{aligned}
& f(z+\omega)-\mu(z) f(z)=0 \\
& \text { is } \prod_{n=1}^{\infty}\left\{\frac{\left[\frac{\Gamma_{1}\left(z+\alpha_{n}\right)}{\Gamma_{1}\left(\alpha_{n}\right)} e^{\left.-\sum_{s=1}^{\rho^{\prime}-1} \psi_{1}^{(s)}\left(\alpha_{n}\right) \frac{z^{s}}{s !}\right]}\left[\frac{\Gamma_{1}\left(\omega+\beta_{n}\right)}{\Gamma_{1}\left(\omega+\beta_{n}-z\right)} e^{\sum_{s=1}^{o^{\prime}-1} \frac{(-z)^{s}}{s !} \psi_{1}^{(s)}\left(\omega+\beta_{n}\right)}\right]\right.}{\left[\frac{\Gamma_{1}\left(z+\gamma_{n}\right)}{\Gamma_{1}\left(\gamma_{n}\right)} e^{-\sum_{s=1}^{\gamma^{\prime}-1} \psi_{1}^{(s)}\left(\gamma_{n} \frac{z^{n}}{s !}\right]}\left[\frac{\Gamma_{1}\left(\omega+\delta_{n}\right)}{\Gamma_{1}\left(\omega+\delta_{n}-z\right)} e^{\sum_{-1}^{\prime-1} \frac{(-z)^{s}}{s !} \psi_{1}^{(s)}\left(\omega+\delta_{n}\right)}\right]\right.}\right\} \\
& \times \exp \left\{\sum_{s=0}^{\delta-2} d_{m} S_{m}(z)\right\},
\end{aligned}
$$

where $\rho^{\prime}, \sigma^{\prime}, \tau^{\prime}, v^{\prime}$ are integers such that $\rho^{\prime}>2 \rho$ and $\rho^{\prime}>2$ and corresponding inequalities, and $\delta$ is the greatest of $\rho^{\prime}, \sigma^{\prime}, \tau^{\prime}, \nu^{\prime}$. The general solution is this meromorphic solution multiplied by a simply periodic function of $z$ of period unity.

The particular solution is a meromorphic function with (1) sequences of zeros proceeding positively from but excluding the points $\beta_{n}$, (2) sequences of zeros proceeding negatively from and including the points $-\gamma_{n}$, (3) sequences of poles proceeding negatively from and including the points $-\alpha_{n}$, (4) sequences of poles proceeding positively from but excluding the points $\delta_{n}$.

10. We have now to consider the solution of the equation

$$
f(z+\omega)-\mu(z) f(z)=0
$$

when $\mu(z)$ is a meromorphic function, as in $\S 9$, except that the Weierstrassian products are of infinite order. The investigation can be briefly indicated: it is an almost obvious extension of the process previously employed. Take first the equation

$$
f(z+\omega)-\phi(z) f(z)=0
$$

where $\phi(z)$ is a similar function to that considered in $\S 4$, but of infinite order. We may put

$$
\phi(z)=\prod_{n=1}^{\infty}\left[\left(1+\frac{z}{\alpha_{n}}\right) e^{\substack{P_{n}-1 \\ s=1} \frac{(-z)^{s}}{s a_{n}^{t}}}\right]
$$

SER. 2. VOL. 2. No. 879 . 
where $\rho_{n}$ is an integer infinite with $n$ such that the series $\sum_{n=1}^{\infty} \frac{1}{a_{n}^{\rho_{n}}}$ is absolutely convergent. (As is well known, it always suffices to take $\rho_{n}=n$.)

Construct the function

$$
P(z)=\prod_{n=1}^{\infty}\left[\frac{\Gamma\left(z+\alpha_{n}\right)}{\Gamma\left(\alpha_{n}\right)} e^{-\sum_{s=1}^{\sigma_{n}-1} \frac{z^{s}}{s !} \psi_{1}^{(s)}\left(\alpha_{n}\right)}\right]
$$

Neglecting a finite number of factors, and taking $|z|<\left|\alpha_{k}\right|$, we see that $P(z)$ converges with

$$
\exp \left[\sum_{n=k}^{\infty} \sum_{s=\sigma_{n}}^{\infty} \sum_{m=0}^{\infty} \frac{(-z)^{s}}{s\left(a_{n}+m \omega\right)^{s}}\right] \text {. }
$$

By exactly the same process as before, we prove that this expression is convergent if $\frac{1}{2} \sigma_{n}>\rho_{n}$ and $\sigma_{n}>2$. The second of these criteria is included in the first, and we see that we must have $\sigma_{n}>2 \rho_{n}$.

Proceed again as before, and we see that

$$
\frac{P(z+\omega)}{P(z)}=\phi(z) e^{f(z)}
$$

where $G(z)$ is an integral function which, when written in the form

$$
G(z)=\sum_{n=0}^{\infty} a_{n} z^{n},
$$

has its coefficients $a$ definite functions of the quantities $\alpha$. Before, then, we can completely solve

we must solve

$$
f(z+\omega)-\phi(z) f(z)=0
$$

$$
f(z+\omega)-e^{G(z)} f(z)=0,
$$

an equation whose solution is $e^{f_{1}(:)}$ where $G_{1}(z)$ is a solution of

$$
f(z+\omega)-f(z)=G(z) .
$$

This equation belongs to the type (B) of $\S 2$. We now proceed to consider it.

11. I have previously investigated * the extended Riemann $\xi$ function, which, when $2 R(z / \omega)$ is positive, can be represented by

$$
\xi(s, z, \omega)=\frac{\iota \Gamma(1-s)}{2 \pi} \int \frac{e^{-y z}}{1-e^{-\omega y}}(-y)^{s-1} d y .
$$


The integral is taken along a contour extending along the axis of $1 / \omega$ from $+\infty / \omega$ round the origin and back again to $+\infty / \omega$, as in the figure, the contour containing no infinities of the subject of integration except the origin. That value of

$$
(-y)^{s-1}=\exp \{(s-1) \log (-y)\}
$$

is taken which has its principal value with respect to the axis to $1 / \omega$.

The function is fundamental in the

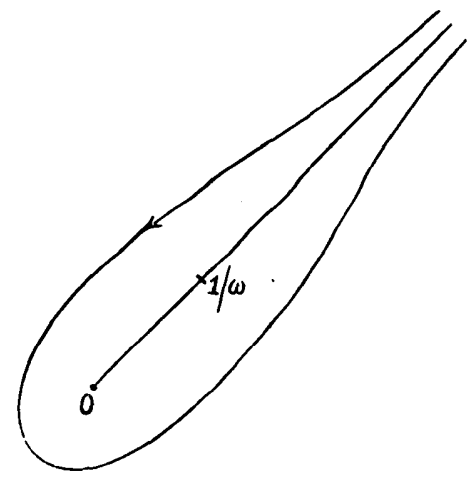
theory of simple gamma and Bernoullian functions, and, as Mellin* has shown, can be represented by a series of functions valid for all values of $z$.

When $s$ is a positive integer greater than 1

$$
\xi(s, z, \omega)=\frac{(-)^{s}}{(s-1) !} \frac{d^{s}}{d z^{s}} \log \Gamma_{1}(z),
$$

and when $s$ is a negative integer

$$
\xi(s, z, \omega)=-\left\{S_{-s}(z)+B_{1-s}(\omega)\right\} .
$$

Also, $q u a$ function of $s, \xi(s, z, \omega)$ is finite for all values of $s$ except $s=1$.

Further,

$$
\xi(s, z+\omega, \omega)-\xi(s, z, \omega)=-z^{-s},
$$

the principal value relative to the axis of $-\omega$ being taken when $s$ is not an integer. Thus for all values, real or complex, of $s$, except $s=1$, $-\xi(-s, z, \omega)$ is a solution of the difference equation

$$
f(z+\omega)-f(z)=z^{s} .
$$

Not only so, but, if $\xi_{k i}(s, z, \omega)$ denotes the function defined as above when $\Varangle R(z / \omega)$ is positive, except that the contour of the integral includes $2 k$ of the poles of the subject of integration besides the origin, $-\xi_{k}(-s, z, \omega)$ is equally a solution of the difference equation (1).

Suppose now that we have the equation

$$
f(z+\omega)-f(z)=G(z)
$$

where $G(z)$ is the integral function $\sum_{n=0}^{\infty} a_{n} z^{n}$. A formal solution will be

$$
-\sum_{n=0}^{\infty} a_{n} \xi_{k_{n}}(-n, z, \omega)
$$

* Mellin, Acta Soc. Sci. Fennice, T. xxrv., No. 10, Part I. Mellin's function is obtained by putting $\omega=1$. I have generalized it and extended the theory to the case of $r$ parameters in my memoir on the multiple gamma function. 
and this solution will be valid provided the series of functions is absolutely convergent at all finite points of the plane. Suppose, in the first place, that $G(z)$ is of finite non-zero order $\rho$. Then ${ }^{*}\left|a_{n}\right|$ behaves when $n$ is very large like $(1 / n !)^{1 / \rho}$ to a first approximation. And therefore

$$
-\sum_{n=0}^{\infty} a_{n} \xi(-n, z, \omega)
$$

behaves like

$$
-\frac{\iota}{2 \pi} \int \frac{e^{-y z}}{1-e^{-\omega y}} \sum_{n=0}^{\infty} n ! a_{n}(-y)^{-n-1} d y=\frac{1}{2 \pi} \int \frac{e^{-y z} \psi(1 / y)}{1-e^{-\omega y}} \frac{d y}{y}
$$

where the contour of integration includes the origin, but no other zero of $1-e^{-\omega z}$, and where

$$
\psi\left(\frac{1}{y}\right)=\sum_{n=0}^{\infty} \frac{(-)^{n} a_{n} n !}{y^{n}} \text {. }
$$

When $\rho<1,\left|(-)^{n} a_{n} n !\right|$ behaves to a first approximation like $(n !)^{-(1-\rho) / \rho}$, and therefore $\psi(z)$ is an integral function of order $\rho /(1-\rho)$. Thus, when $\rho<1$, a solution of

is

$$
\begin{gathered}
f(z+\omega)-f(z)=G(z) \\
\frac{\iota}{2 \pi} \int \frac{e^{-y z z} \psi(1 / y)}{1-e^{-\omega y}} \frac{d y}{y} .
\end{gathered}
$$

This integral defines a solution for all values of $z$ and $\omega$. For the integral taken along the prescribed contour may be at once reduced to an integral taken round a circle, centre the origin, and radius less than $|2 \pi i / \omega|$.

12. We have thus found a solution of

$$
f(z+\omega)-f(z)=G(z)
$$

where the order of $G(z)$ is less than unity.

If the order of $G(z)$ is not less than unity, one of two things may happen : $\psi(z)$ may still be a function convergent within a circle of finite radius $\lambda$, or the series for $\psi(z)$ may diverge absolutely. In the first case our course is evident: we take the previous integral round a circle of radius $>1 / \lambda$, and thus obtain a solution of the difference equation. In the second case the series for $\psi(y)$ is truly asymptotic, i.e., of zero radius of convergence, and the solution breaks down. But in this case the formal solution which we have found is not altogether nugatory. For $G(z)$ and $\psi(z)$ are what Borel $\dagger$ has called associated functions, and, as may be

"See the author's "Classification of Integral Functions" (loc. cit. $\$ 3), \S 35$, p. 349, where references to the work of Hadamard, Borel, and othors are given.

† Borel, Annales de l'Ecole Normale Supérieure (1899), pp: 1-136, especially pp. 89 et seq. 
deduced from the theory of asymptotic series, ${ }^{*}$ the divergent series for $\psi(y)$ may be used to determine a function which possesses the properties which are required to build up by the method indicated a solution of the difference equation.

By means, however, of a series of modified functions $\xi_{n}(-m, z, \omega)$ we may formally give a convergent expansion for this solution.

Let $\zeta_{n}(-m, z, \omega)$ denote the integral

$$
\frac{\iota m !}{2 \pi} \int \frac{e^{-z y}}{1-e^{-\omega y}} \frac{d y}{(-y)^{m+1}}
$$

taken round a circle of radius $(2 m+1) \pi / / \omega$, so that the subject of integration has $2 m$ poles inside the contour in addition to the origin. At the pole $2 k \pi i / \omega$. the residue is

$$
\frac{-m ! e^{-2 k \pi \varepsilon ! \omega}}{\omega\left(-\frac{2 k \pi \iota}{\omega}\right)^{m+1}} .
$$

The function $\xi_{m}(-m, z, \omega)$ therefore differs from $\xi(-m, z, \omega)$ by a function of $z$ which is simply periodic of period $\omega$. We have

$$
\xi_{n}(-m, z+\omega, \omega)-\xi_{m}(-m, z, \omega)=-z^{m},
$$

and hence $-\sum_{n=0}^{\infty} a_{n} \xi_{n}(-n, z, \omega)$ is a formal solution of the difference equation

$$
f(z+\omega)-f(z)=G(z) .
$$

Now, when $n$ is large, $\dagger$

$$
\left|\xi_{n}(-n, z, \omega)\right|<\frac{n ! e^{\left|z \frac{(2 n+1) \pi}{\omega}\right|} \mid}{K\left|(2 n+1) \frac{\pi l}{\omega}\right|^{n}},
$$

where $K$ is the minimum value of $\left|1-e^{-\omega y}\right|$ on the circle of radius $(2 n+1) \pi \imath / \omega$, and is therefore $>0$.

Thus

$$
\left|\xi_{n}(-n, z, \omega)\right|<\frac{e^{\left|z \frac{(2 n+1) \pi}{\omega}\right|}}{K\left|\frac{2 \pi \iota}{\omega} e\right|^{n}} \text { approximately. }
$$

Now, since $G(z)$ is an integral function, $\left|\sqrt[n]{ } a_{n}\right|$ tends to zero with $1 / n$.

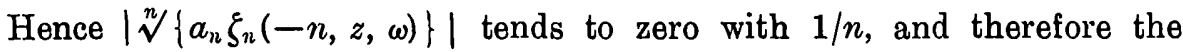
formal solution $\sum_{n=0}^{\infty} a_{n} \xi_{n}(-n, z, \omega)$ is a series of functions absolutely convergent for all finite values of $|z|$. It therefore represents an integral

* See the author's Memoir on Integral Functions, § 29, \&c.

+ Forsyth, Theory of Functions (1900), \$15. 
function of $z$. Therefore there exists a solution of

$$
f(z+\omega)-f(z)=G(z),
$$

where $G(z)$ is any integral function of $z$, which is an integral function: the general solution is obtained by adding to this particular solution an arbitrary simply periodic function of $z$ of period $\omega$.

The substitution of the function $\xi_{n}(-n, z, \omega)$ for $\xi(-n, z, \omega)$ is substantially the same as the process employed by Mittag-Leffler in his well known theorem. It is from this point of view that the matter has been considered by Hurwitz, who has anticipated the results just obtained.

13. We see now that we have the means of completing our solution of the equation

$$
f(z+\omega)-\mu(z) f(z)=0
$$

for all cases in which $\mu(z)$ is a meromorphic function of infinite order with simple sequences of non-repeated zeros and poles.

A particular solution is a meromorphic function of $z$ which may be expressed in the form

$$
e^{G_{1}(z)} \prod_{n=1}^{\infty}\left[\frac{\mathbb{f}_{1, n}(z)}{\sqrt{\mathbb{F}_{2, n}(z)}} \frac{\sqrt{\mathbb{F}_{3, n}(z)}}{\sqrt{\mathfrak{F}_{4, n}(z)}}\right],
$$

where $\mathbb{N}(z)$ denotes a primary gamma factor, and where $G_{1}(z)$ is an integral function which reduces to a polynomial when the orders of the integral functions $\phi(z)$ and $\chi(z)$ whose quotient $\frac{\phi(z)}{\chi^{(z)}}$ forms $\mu(z)$ are finite.

The products

$$
\prod_{n=1}^{\infty}\left[\mathfrak{H}_{r, n}(z)\right] \quad(r=1,2,3,4)
$$

are constructed from the integral functions $\phi(z)$ and $\chi(z)$. In such construction there is an element of arbitrariness as regards the finite zeros of $\phi(z)$ and $\chi(z)$, correspondining to the fact that, since

$$
\Gamma_{1}(z) \Gamma_{1}(\omega-z)=\frac{\pi}{\omega \sin \pi z / \omega},
$$

we may take either $\Gamma_{1}(z)$ or $\frac{1}{\Gamma_{1}(\omega-z)}$ as the basis of the primary factor. But ultimately the infinite terms of the products must be such that, corresponding to zeros of $\phi(z)$ and $\chi(z)$ negative with regard to $\omega$, we must have sequences of poles and zeros respectively of the solution negatively congruent to and including these zeros, while, corresponding to positive zeros of $\phi(z)$ and $\chi(z)$, we must have sequences of zeros and 
poles respectively which are positively congruent to but exclude the corresponding points.

From a diagram the rule for congruent zeros is evident: the sequences from zeros or poles of $\mu(z)$ whose moduli are very large must not cross the finite part of the plane. And this geometrical point of view explains the restriction introduced by the analysis; if this phenomenon did occur, we should get, in general, eritical points of the solution at all points in the finite part of the plane, so that this part of the plane would be a lacunary space for the function. In special cases the distribution of the zeros and poles of $\mu(z)$ may be such that they lie on a finite number of lines parallel to the axis of $\omega$, and then we should get lines of singularity in the finite part of the plane; except in the case when these sets of zeros and poles are all congruent with regard to $\omega$, when isolated essential singularities would arise.

The previous investigation may be readily extended to cases when $\mu(z)$ has repeated zeros or poles or is of multiple sequence. After the previous investigation a discussion of these cases would be tedious : a single example will be given later.

14. It is possible to express the principal solution of

$$
f(z+\omega)-\mu(z) f(z)=0
$$

in two other forms. We proceed first to write it as a doubly infinite product of strict Weierstrassian form. As before, we express $\mu(z)$ as a quotient of two pairs of integral functions, each with its ultimate sequence of zeros all positive or all negative with regard to $\omega$, so that a typical factor is the function

defined as in $\S 10$.

$$
\phi_{1}(z)=\prod_{n=1}^{\infty}\left[\left(1+\frac{z}{a_{n}}\right) e^{\left.\sum_{n=1}^{p_{n}-1} \frac{(-z)^{s}}{s a_{n}^{s}}\right]},\right.
$$

The solution of

$$
f(z+\omega)-\phi_{1}(z) f(z)=0
$$

has been shown $(\$ 10)$ to be

$$
P_{1}(z)=e^{G_{1}(z)} \prod_{n=1}^{\infty}\left[\frac{\Gamma\left(z+a_{n}\right)}{\Gamma\left(a_{n}\right)} e^{-\sum_{s=1}^{\sigma_{n}-1} z^{s} \psi_{i}^{\left(s_{1}\right)\left(a_{n}\right)}}\right],
$$

where $\sigma_{n}>2 \rho_{n}$ when $\rho_{n}$ depends upon $n$, and where $\sigma>2 \rho$ and $\sigma>2$ when $\rho$, the integer next greater than the order of $\phi_{1}(z)$, is finite.

Consider now the product

$$
Q_{1}(z)=\prod_{n=1}^{\infty} \prod_{m=0}^{\infty}\left[\left(1+\frac{z}{\alpha_{n}+m \omega}\right) e^{\sum_{s=1}^{\tau_{n}-1} \frac{1}{k}\left(\frac{-z}{\alpha_{n}+m \omega}\right)^{s}}\right] .
$$


It converges with

$$
\exp \left[\sum_{n}^{\infty} \sum_{m}^{\infty} \sum_{s=\tau_{n}}^{\infty}-\frac{1}{s}\left(\frac{-z}{a_{n}+m \omega}\right)^{s}\right],
$$

those terms (finite in number) being omitted from the summation with regard to $n$ and $n$ for which $\left|a_{n}+m \omega\right|<|z|$.

By an extension of the process employed in $\S \S 4$ and 5 , we see that this expression is convergent if $\tau_{n}>2 \rho_{n}$ (a detailed proof for a more complicated case is given in $\S 16$ ). If, then, we put $\tau_{n}=\sigma_{n}$, we see that $P_{1}(z) Q_{1}(z)$ is a function with no zeros or poles, and therefore of the form $e^{G(z)}$ where $G(z)$ is an integral function. Therefore the principal solution of

may be written

$$
f(z+\omega)-\phi_{1}(z) f(z)=0
$$

$$
e^{G_{1}(z)} / \prod_{n=1}^{\infty} \prod_{m=0}^{\infty}\left[\left(1+\frac{z}{a_{n}+m \omega}\right) e^{\sum_{s=1}^{\sigma_{n}-1} \frac{1}{s}\left(\frac{-z}{a_{n}+m \omega}\right)^{s}}\right] .
$$

A solution of

$$
f(z+\omega)-\phi_{2}(z) f(z)=0
$$

where

$$
\phi_{2}(z)=\prod_{n=1}^{\infty}\left[\left(1-\frac{z}{\beta_{n}}\right) e^{\sum_{s=1}^{\rho_{n}-1} \frac{1}{s}\left(\frac{z}{\beta_{n}}\right)^{s}}\right]
$$

and the real parts of $\beta_{1} / \omega, \ldots, \beta_{n} / \omega, \ldots$ are positive is, similarly,

$$
\left.e^{G_{2}(z)} \prod_{n=1}^{\infty} \prod_{m=1}^{\infty}\left[\left(1-\frac{z}{m \omega+\beta_{n}}\right) e^{\sum_{s=1}^{\sigma_{n}-1} \frac{1}{s}\left(-2 \overline{\beta_{n}}+\overline{m \omega}\right.}\right)^{s}\right]
$$

where $\sigma_{n}>2 \rho_{n}$ if $\rho_{n}$ depends upon $n$, and $\sigma_{n}>2 \rho$ and $\sigma>2$ if $\phi_{2}(z)$ is of finite order. Solutions of

$$
f(z+\omega)-f(z) / \chi_{1}(z)=0, \quad f(z+\omega)-f(z) / \chi_{2}(z)=0
$$

can be written down in similar manner, and the principal solution of

$$
f(z+\omega)-\mu(z) f(z)=0
$$

will be the product of all four of such solutions.

We see that the products $Q_{1}(z), \ldots$ which arise are, in the notation to which reference was made in $\S 3$, functions of double sequence, linear with respect to one of the sequences. If $\sum_{n=1}^{\infty} \sum_{n=0}^{\infty} \frac{1}{\left|\left(a_{n}+m \omega\right)\right|^{R+e}}$ is convergent and $\sum_{n=1}^{\infty} \sum_{n=0}^{\infty} \frac{1}{\left|\left(a_{n}+m \omega\right)\right|^{R-e}}$ is divergent, we say that the order of the function is $R$, this being the natural extension of the definition of order for functions of double linear sequence. With this definition we see $(\$ 5)$ that the order of $Q_{1}(z)$ is at most 2 or $2 \rho^{\prime}$, where $\rho^{\prime}$ is the order 
of the function $\phi_{1}(z)$ from which $Q_{1}(z)$ is derived, whichever be the greater of these two quantities.

15. We proceed to write the solution just obtained in yet another form. Take, as before, the equation

$$
f^{\prime}(z+\omega)-\phi_{1}(z) f(z)=0
$$

and consider the product

$$
R_{1}(z)=\prod_{m=0}^{\infty}\left[\frac{\phi_{1}(m \omega)}{\phi_{1}(z+m \omega)} e^{\tau_{m=1}^{\tau_{m-1}} z_{s !}^{s} x_{1}^{(j)}(m \omega)}\right]
$$

where

$$
\chi_{1}^{(r)}(z)=\frac{d^{r}}{d z^{r}} \log \phi_{1}(z) .
$$

Since $\alpha_{n}$ is positive with respect to $\omega$, we have $\left|\alpha_{n}+m \omega\right|>|m \omega|$, and therefore, for values of $z$ inside a circle centre the origin and radius $|k \omega|$, $\log \phi_{1}(z+m \omega)$, where $m>k$, can be expanded in an absolutely convergent series of powers of $z$. Hence each term of $R_{1}(z)$ after the first $k$ terms can for such values of $z$ be written in the form

$$
\exp \left[-\sum_{s=\tau_{n}}^{\infty} \frac{z^{s}}{s !} \chi_{1}^{(s)}(m \omega)\right]
$$

We may choose $\tau_{m}$ so that the series inside the bracket is as small as we please, let us say $<\epsilon_{m}$. We choose the $\tau^{\prime}$ 's so that $\sum_{m=k}^{\infty} \epsilon_{m}$ is absolutely convergent, and thus ensure the convergency of $R_{1}(z)$.

But $R_{1}(z)$ has the same poles as $P_{1}(z)$, and neither function has any finite zeros. Hence the solution of

may be written

$$
f(z+\omega)-\phi_{1}(z) f(z)=0
$$

$$
e^{\sigma_{1}(z)} \prod_{m=0}^{\infty}\left[\frac{\phi_{1}(m \omega)}{\phi_{1}(z+m \omega)} e^{\sum_{s=1}^{\tau_{m}-1} z^{s} z^{s} x_{1}^{(s)}(m \omega)}\right]
$$

where $G_{1}(z)$ is an integral function of $z$.

We may now prove that it is sufficient to take $\tau_{m}$ equal to the quantity $\sigma$ of $\S 6$ in the case when the order of $\phi_{1}(z)$ is a finite quantity.

For, for values of $z$ such that $|z|<|k \omega|$, the product $R_{1}(z)$ is convergent with

Now

$$
\exp \left[-\sum_{m=k}^{\infty} \sum_{s=\tau_{n}}^{\infty} \frac{z^{s}}{s !} \chi_{1}^{(s)}(m \omega)\right]
$$

$$
\chi_{1}^{(s)}(z)=\sum_{n=1}^{\infty}\left[\frac{(-)^{s-1}(s-1) !}{\left(z+a_{n}\right)^{s}}+(-)^{s} \sum_{r=0}^{\rho_{n}-s-1} \frac{(r+1)(r+2) \ldots(r+s-1)(-z)^{r}}{a_{n}^{r+s}}\right] ;
$$


so that $R_{1}(z)$ is convergent with

$$
\sum_{m=k}^{\infty} \sum_{s=\tau_{m}}^{\infty} \sum_{n=1}^{\infty} \frac{(-)^{s} z^{s}}{s}\left[\frac{1}{\left(m \omega+\alpha_{n}\right)^{s}}-\sum_{r=0}^{\rho_{n}-s-1}\left(\begin{array}{c}
r+s-1 \\
s-1
\end{array}\right) \frac{(-m \omega)^{r}}{a_{n}^{r+s}}\right] .
$$

When $\rho_{n}$ is finite and equal to $\rho$, the integer next greater than the finite order of $\phi_{1}(z)$, the summation in the bracket vanishes when $s>\rho$. The series last written becomes

$$
\sum_{m=k}^{\infty} \sum_{s=\tau_{m}}^{\infty} \sum_{n=1}^{\infty} \frac{(-)^{s} z^{s}}{s\left(a_{n}+m \omega\right)^{s}}
$$

and will be convergent when $|z|<|\sqrt{ }(k \omega)|$ if we take $\tau_{m}=\sigma$ (a quantity independent of $m$ ) and such that $\sigma>2 \rho$ and $\sigma>2$. For in this case the series of moduli is

$$
\begin{aligned}
& <\sum_{m=k}^{\infty} \sum_{s=\sigma}^{\infty} \sum_{n=1}^{\infty} \frac{|z|^{s}}{s|m \omega|^{\beta s}} \frac{1}{\left|a_{n}\right|^{\beta s}} \\
& <\frac{1}{\sigma} \sum_{m=k}^{\infty} \frac{|z|^{\sigma}}{(m(\omega))^{j \sigma} ! 1-\left|\frac{z}{\sqrt{ }(m \omega)}\right| j_{j}} \sum_{s=\sigma}^{\infty} \sum_{n=1}^{\infty} \frac{1}{\left|\alpha_{n}\right|^{\beta s}},
\end{aligned}
$$

provided $|z|<|\sqrt{ }(k \omega)|$. And therefore within the circle defined by the inequality last written the product is absolutely convergent provided $\sigma>2 \rho$ and $\sigma>2$. Since we may make $|\sqrt{ }(k \omega)|$ as large as we please, the product must be, when $\tau_{\mu h}=\sigma$, absolutely convergent for all finite values of $|z|$.

16. As has been stated, the investigation of the solution of

$$
f(z+\omega)-\mu(z) f(z)=0
$$

can be extended to cases when $\mu(z)$ is a function with repeated zeros or poles or of multiple sequence. Suppose, as an example, that

$$
\mu(z)=\prod_{n=1}^{\infty}\left[\left(1+\frac{z}{a_{n}}\right)^{\mu_{n}} \exp \left\{\mu_{n} \sum_{s=1}^{\sigma_{n}-1} \frac{1}{s}\left(-\frac{z}{a_{n}}\right)^{s}\right\}\right] ;
$$

so that $\mu(z)$ is a function with repeated zeros. The function will be of finite order $\rho$ if we can choose for $\sigma_{n}$ a number $\sigma$ independent of $n$ and such that $\sigma>\rho>\sigma-1$, where $\Sigma \frac{\mu_{n}}{\left|a_{n}\right|^{\rho+e}}$ is convergent and $\Sigma \frac{\mu_{n}}{\left|a_{n}\right|^{\rho-e}}$ is divergent, however small the positive quantity $\epsilon$ may be. In other cases we must take $\sigma_{n}$ such that $\sum_{n=k}^{\infty} \mu_{n}\left|\frac{z}{a_{n}}\right|^{\sigma_{n}}$ is convergent when $|z|$ is finite. Since the sequence $\alpha_{0}, \alpha_{1}, \ldots, \alpha_{n}, \ldots$ tends to infinity, this is equivalent to saying that $\sum_{n=i}^{\infty} \frac{\mu_{n}}{\left|a_{n}\right|^{\sigma_{n}}}$ must be convergent.

If now the quantities $\alpha_{n}$ be positive with respect to $\omega$, we may, to obtain a solution corresponding to the second of the three preceding 
types, form the product

$$
\prod_{n=1}^{\infty} \prod_{n=0}^{\infty}\left[\left(1+\frac{z}{a_{n}+m \omega}\right)^{\mu_{n}} \exp \left\{\mu_{n} \sum_{s=1}^{\tau_{n}-1} \frac{1}{s}\left(\frac{-z}{a_{n}+m \omega}\right)^{s}\right\}\right] .
$$

This product will be convergent for values of $z$ such that $|z|$ is sufficiently small if $\exp \left[-\sum_{n}^{\infty} \sum_{m}^{\infty} \sum_{s=\tau_{n}}^{\infty} \frac{\mu_{n}}{s}\left(\frac{-z}{a_{n}+m \omega}\right)^{s}\right]$ is convergent, those terms (finite in number) being onitted from the summations with regard to $m$ and $n$ for which $\left|\alpha_{n}+m \omega\right|<|z|$. The series is convergent with

$$
\sum_{n}^{\infty} \sum_{m}^{\infty} \frac{\mu_{n}\left|\frac{z}{a_{n}+m \omega}\right|^{\tau_{n}}}{1-\left|\frac{z}{a_{n}+m \omega}\right|} ;
$$

and therefore with

$$
\sum_{n}^{\infty} \sum_{m}^{\infty} \mu_{n}\left|\frac{z}{\alpha_{n}+m \omega}\right|^{\tau_{n}}
$$

Suppose now that $|l \omega|>|z|>|(l-1) \omega|, \quad\left|\alpha_{k}\right|>|z|>\left|\alpha_{k-1}\right|$.

Then the double series is less than

$$
\sum_{n=0}^{k-1} \sum_{m=0}^{\infty}+\sum_{m=0}^{l-1} \sum_{n=0}^{\infty}+\sum_{m=l}^{\infty} \sum_{n=k}^{\infty} \mu_{n}\left|\frac{z}{\alpha_{n}+m \omega}\right|^{\tau_{n}} \text {. }
$$

The first set of single series is finite for all finite values of $k$ if $\tau_{n}>1$ for all values of $n$. The second set of series is finite for all finite values of $l$ if $\tau_{n}>\sigma_{n}$. The third double series is less than $(\$ 5)$

$$
\sum_{n=l}^{\infty} \sum_{n=k}^{\infty} \mu_{n} \frac{|z|^{\tau_{n}}}{\left|2 u_{n}\right|^{1 \tau_{n}}|m \omega|^{\frac{\beta}{\tau_{n}}}}<\sum_{m=i}^{\infty}\left|\frac{z}{m \omega}\right|^{\mid \tau_{k}} \sum_{n=k}^{\infty} \mu_{n}\left|\frac{z}{2 \alpha_{n}}\right|^{\dagger \tau_{n}},
$$

where $\tau_{k}$ is the value of $\tau_{n}$ when $n=k$, this value being less than any succeeding value for sufficiently large values of $k$. Thus the third double series is convergent if $\tau_{k}>2, \tau_{n}>2 \sigma_{n}$. Finally, then, the original product is absolutely convergent for all finite values of $|z|$ if $\tau_{n}>2 \sigma_{n}$, $\tau_{n}$ being $>1$ for all values of $n$. When $\sigma_{n}$ is independent of $n^{*}$ and equal to $\sigma$, say, we must have $\tau>2 \sigma$ and $\tau>2$.

The reciprocal of the product multiplied by a function of the type $e^{G_{1}(i)}$ is a solution of the difference equation.

The method of extension to cases of more complicated integial or meromorphic functions is now obvious.

We have the general theorem that a solution of the linear difference equation

$$
f(z+\omega)-\mu(z) f(z)=0
$$


where $\mu(z)$ is a meromorphic function of $z$ can always be obtained as a meromorphic function with sequences of poles and zeros of the same order as the corresponding poles or zeros of $\mu(z)$. This solution can be written in three different forms. In each the sequences of zeros proceed positively from but exclude the positive zeros of $\mu(z)$ and negatively from and include the negative poles of $\mu(z)$, while the sequences of poles proceed positively from but exclude the positive poles of $\mu(z)$ and negatively from and include the negative zeros of $\mu(z)$.

17. We have now to consider equations of the type $B(\S 2)$, that is an equation

$$
f(z+\omega)-f(z)=\mu(z)
$$

where $\mu(z)$ is a meromorphic function of $z$.

For this purpose we express $\mu(z)$ by Mittag-Leffler's theorem, just as to solve

$$
f(z+\omega)-\mu(z) f(z)=0
$$

we expressed $\mu(z)$ as a quotient of Weierstrassian products.

Briefly recapitulated Mittag-Leffler's process is as follows. When the poles of $\mu(z)$ are arranged in order of non-decreasing moduli, let the $r$-th pole $a_{r}$ be of order $k_{r}$ and let the expansion of $\mu(z)$ in its vicinity be

$$
\begin{aligned}
\mu(z) & =\sum_{s=1}^{l_{s}} \frac{a_{s}}{\left(z-a_{r}\right)^{s}}+b_{0}+\sum_{s=1}^{\infty} b_{s}\left(z-\alpha_{r}\right)^{s} \\
& =\log _{r}\left(z-\alpha_{r}\right)+b_{0}+\sum_{s=1}^{\infty} b_{s}\left(z-a_{r}\right)^{s} \text {, say. }
\end{aligned}
$$

So long as $|z|<\left|\alpha_{r}\right|$ we have the expansion

$$
q_{r}\left(z-\alpha_{r}\right)=\sum_{m=0}^{\infty}{ }_{r} c_{m} z^{m}
$$

Take now $\quad F_{r}(z)=\mathscr{Q}_{r}\left(z-\alpha_{r}\right)-\sum_{m=0}^{m_{r}-1}{ }_{r} c_{m} z^{m} ;$

so that, when $|z|<\left|\alpha_{r}\right|$,

$$
F_{r}(z)=\sum_{m=m_{r}}^{\infty}{ }_{r} c_{m} z^{m}
$$

Choose now a value of $m_{r}$ sufficiently large and we shall have, when $|z|<\left|a_{r}\right|,\left|F_{r}(z)\right|<\epsilon_{r}$, where the series $\sum_{r=1}^{\infty} \epsilon_{r}$ is absolutely convergent. And now $\mu(z)=\sum_{r=0}^{\infty} F_{r}(z)+G(z)$ where $G(z)$ is an integral function of $z$.

To solve the equation $f(z+\omega)-f(z)=\mu(z)$, 
divide the poles of $\mu(z)$ into two sets $\alpha_{0}, \alpha_{1}, \ldots, \alpha_{n}, \ldots$ and $\beta_{0}, \beta_{1}, \ldots, \beta_{n}, \ldots$ respectively negative and positive with respect to $\omega$.

We know that $\frac{(-)^{s-1}}{(s-1) !} \psi_{1}^{(s)}(z)$ is a solution of

$$
f(z+\omega)-f(z)=\frac{1}{z^{s}},
$$

and therefore we take a function $\phi_{r}^{\prime}(z)$ corresponding to $\mathbb{2}_{r}\left(z-\alpha_{r}\right)$, and such that

$$
\phi_{r}(z)=\sum_{s=1}^{k_{r}} \frac{(-)^{s-1}}{(s-1) !} a_{s} \psi_{1}^{(s)}\left(z-\alpha_{r}\right)
$$

Then, since $\left|m \omega-\alpha_{r}\right|>\left|\alpha_{r}\right|$, we shall have, when $|z|<\left|\alpha_{r}\right|$, the expansion

$$
\phi_{r}(z)=\sum_{m=0}^{\infty}{ }_{r} C_{m} z^{m}
$$

18. We now need the following lemma:-

If $\phi(z)$ be a solution of the difference equation

$$
f(z+\omega)-f(z)=\boldsymbol{q}(z-\alpha),
$$

where $(z-\alpha)$ can, within a circle of radius $|\alpha|$ and centre the origin, be expanded in a convergent series $\sum_{m=0}^{\infty} c_{m} z^{m}$, so that when $m$ is large to a first approximation $\left|c_{m}\right|=1 /|\alpha|^{m}$, then, if $|\alpha(2 \pi \iota / \omega)|>1 / e$, we shall have, within a circle of radius $\rho$ given by

$$
e^{\rho|2 \pi \iota / \omega|}=e|\alpha|\left|\frac{2 \pi \iota}{\omega}\right|,
$$

an expansion for $\phi(z)$ of the form

$$
\varpi(z, \alpha, \omega)-\sum_{m=0}^{\infty} c_{m} \xi_{m}(-m, z, \omega),
$$

the series being absolutely convergent so long as $|z|<\rho$ and $\varpi(z, \alpha, \omega)$ being a simply periodic function of $z$ of period $\omega$.

Consider the series $\sum_{m=0}^{\infty} c_{m} \xi_{m}(-m, z, \omega)$. By the results of $\S 12$ it is convergent, provided

$$
e^{\left|z\left(2 \pi \iota^{\prime} \omega\right)\right|}<\left|\frac{2 \pi \iota e \alpha}{\omega}\right|
$$

i.e., provided $|z|<\rho$, as above defined.

We note that $\rho$ always exists as a positive quantity provided $|\alpha(2 \pi \iota / \omega)|>1 / e$. Again $\rho$ is less than $|\alpha|$. For, putting $|2 \pi \iota / \omega|=\theta, \rho$ is 
defined by the equality

$$
e^{\rho \theta-1}=|\alpha| \theta .
$$

Now, if $x$ be real and positive, or real, negative, and $<1$,

therefore

$$
\begin{gathered}
e^{x}>1+x ; \\
\rho<|\alpha| .
\end{gathered}
$$

Further, we note that $\rho$ increases to infinity with $a$.

Assume now that $|\alpha|>e^{-1}|\omega / 2 \pi \iota|$. Then, within a circle of radius $\rho, \sum_{m=0}^{\infty} c_{m} \xi_{m}(-m, z, \omega)$ is convergent. Therefore within this circle

$$
\sum_{m=0}^{\infty} c_{m} \xi_{m}(-m, z, \omega)-\sum_{m=0}^{\infty} c_{m} z^{n}=\sum_{m=0}^{\infty} c_{m} \xi_{m}(-m, z+\omega, \omega)
$$

is convergent. Hence, provided $|z|<\rho, \sum_{m=0}^{\infty} c_{n l} \xi_{m n}(-m, z, \omega)$ is a solution of the difference equation

or

$$
\begin{aligned}
& f(z+\omega)-f(z)=\sum_{m=0}^{\infty} c_{m} z^{m}, \\
& f(z+\omega)-f(z)=\text { ( }(z-\alpha) .
\end{aligned}
$$

And the general solution will be

$$
\varpi(z, \alpha, \omega)-\sum_{m=0}^{\infty} c_{m} \xi_{m}(-m, z, \omega),
$$

where $\varpi(z, \alpha, \omega)$ is a simply periodic function of $z$ of period $\omega$.

We note that, if $\phi(z)$ has no singularities within the circle $|z|<\rho$, neither has $\varpi(z, \alpha, \omega)$ singularities within the same circle. The lemma is thus established.

Corollary. - We can deduce an interesting result in the theory of the expansion of an arbitrary function in a series of functions.

Let $f(z)$ be an arbitrary integral function. Then $f(z+\omega)-f(z)$ is an integral function and therefore capable of expansion in the form

$$
\sum_{m=0}^{\infty} c_{m} z^{m}
$$

The general solution of the equation

$$
f(z+\omega)-f(z)=\sum_{m=0}^{\infty} c_{m} z^{m}
$$

is

$$
\varpi(z, \omega)-\sum_{m=0}^{\infty} c_{m} \xi_{m}(-m, z, \omega),
$$

the series being valid for all finite values of $|z|$. 
Hence any integral function of $z$ can be expanded in the form

$$
\varpi(z, \omega)-\sum_{m=0}^{\infty} c_{m} \xi_{m}(-m, z, \omega)
$$

where $\varpi(z, \omega)$ is a finite simply periodic function of $z$ with no finite poles, and the expansion will hold for all finite values of $|z|$.

19. Take now the function

$$
\phi_{r}(z)=\sum_{s=1}^{k_{r}} \frac{(-)^{s-1}}{(s-1) !} a_{s} \psi_{1}^{(s)}\left(z-\alpha_{r}\right) .
$$

If $\left|\alpha_{r}(2 \pi \iota / \omega)\right|>1 / e$, which will be the case for sufficiently large values of $r$, since $\mu(z)$ is meromorphic and consequently the sequence of its poles tends to infinity, we may expand $\phi_{r}(z)$ in the form

$$
\varpi_{r}\left(z, \alpha_{r}, \omega\right)-\sum_{m=0}^{\infty}{ }_{r} c_{m} \xi_{m}(-m, z, \omega),
$$

the expansion being valid, provided $|z|<\rho_{r}$, where $\rho_{r}$ tends to infinity with $\left|\alpha_{r}\right|$ and therefore with $r$.

Let us take

$$
\Phi_{r}(z)=\phi_{r}(z)-\varpi_{r}\left(z, a_{r}, \omega\right)+\sum_{m=0}^{\mu_{r}-1}{ }_{r} c_{m} \xi_{m n}(-m, z, \omega) ;
$$

then, so long as $|z|<\rho_{r}$, we have

$$
\Phi_{r}(z)=-\sum_{m=\mu_{r}}^{\infty}{ }_{r} c_{m} \xi_{m}(-m, z, \omega),
$$

and the modulus of this series may for sufficiently large values of $\mu_{r}$ be made as small as we please.

We choose $\mu_{r}$ such that, when $|z|<\rho_{r},\left|\Phi_{r}(z)\right|<\epsilon_{r}$, where $\sum_{r=1}^{\infty} \epsilon_{r}$ is absolutely convergent.

Then, for values of $|z|<\rho_{r}$, the series $\sum_{s=r}^{\infty} \Phi_{s}(z)$ is absolutely convergent, and hence $\sum_{s=1}^{\infty} \Phi_{s}(z)$ is a series convergent at all points of the plane except the points negatively congruent to and including the poles $a_{r}$. And we have

$$
\Phi_{r}(z+\omega)-\Phi_{r}(z)=\sum_{s=1}^{k_{r}} \frac{a_{s}}{\left(z-\alpha_{r}\right)^{s}}-\sum_{n=0}^{\mu_{r}-1}{ }_{n} c_{m l} z^{m}=\sum_{n=\mu_{r}}^{\infty}{ }_{r} c_{m} z^{m},
$$

by $\$ 17$, when $|z|<\alpha_{r}$. 
20. For the poles $\beta_{r}$ of $\mu(z)$ we proceed in an analogous manner.

By the fundamental difference equation for the gamma functions $\psi_{1}^{(s)}(\omega-z) /(s-1) !$ is a solution of $f(z+\omega)-f(z)=1 / z^{s}$, when $s$ is an integer.

If near its $r$-th pole $\beta_{r}$, positive with regard to $\omega, \mu(z)$ admit the expansion

$$
\mu(z)=\sum_{s=1}^{l_{r}} \frac{b_{s}}{\left(z-\beta_{r}\right)^{s}}+e_{0}+e_{1}\left(z-\beta_{r}\right)+\ldots,
$$

we construct the function

$$
\psi_{r}(z)=\sum_{s=1}^{l_{r}} \frac{b_{s}}{(s-1) !} \psi_{1}^{(s)}\left(\omega+\beta_{r}-z\right)
$$

Then, when $|z|<\sigma_{r}$, where $\sigma_{r}$ tends to infinity with $\beta_{r}$, we have the absolutely convergent expansion

$$
\psi_{r}(z)=\varpi\left(z, \beta_{r}, \omega\right)-\sum_{m=0}^{\infty}{ }_{r} d_{m} \xi_{m}(-m, z, \omega) .
$$

We take $\quad \Psi_{r}(z)=\psi_{r}(z)-\varpi\left(z, \beta_{r}, \omega\right)+\sum_{m=0}^{\nu_{r}-1}{ }_{r} d_{m} \xi_{m}(-m, z, \omega)$,

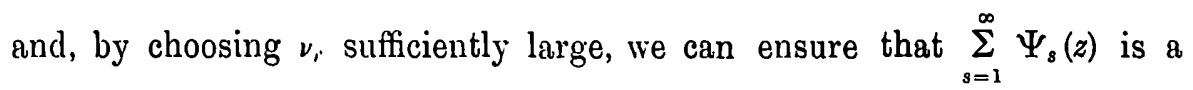
series convergent at all points of the plane, except the points positively congruent to but not including the poles $\beta_{r}$.

And now $\sum_{s=1}^{\infty}\left[\Phi_{s}(z)+\Psi_{s}(z)\right]$ will be a meromorphic function satisfying the difference equation

$$
f(z+\omega)-f(z)=\mu_{1}(z),
$$

where $\mu_{1}(z)-\mu(z)$ is an integral function which may be expanded in the series $\sum_{m=0}^{\infty} e_{m} z^{n}$, valid for all finite values of $|z|$.

Finally, then, a solution of the equation $f(z+\omega)-f(z)=\mu(z)$ is given by

$$
\sum_{s=1}^{\infty}\left[\Phi_{s}(z)+\Psi_{s}(z)-e_{s} \xi_{s}(-s, z, \omega)\right]
$$

and the general solution is this function plus an arbitrary simply periodic function of $z$ of period $\omega$.

The form of the principal solution just obtained can be modified as regards sequences proceeding from the poles of $\mu(z)$, which are in the finite part of the plane. 
21. It is possible to give two other forms to the solution just obtained. Retaining the notation of $\S 20$, consider $\phi_{r}(z)$. We have

$$
\begin{aligned}
& \phi_{r}(z)= \sum_{s=1}^{k_{r}} \frac{(-)^{s-1}}{(s-1) !} a_{s} \psi_{1}^{(s)}\left(z-\alpha_{r}\right) \\
&=-\sum_{s=2}^{k_{r}} a_{s} \sum_{m=0}^{\infty} \frac{1}{\left(z-\alpha_{r}+m \omega\right)^{s}} \\
& \quad \quad-a_{1}\left\{\frac{\gamma-\log \omega}{\omega}+\frac{1}{z-\alpha_{r}}+\sum_{m=1}^{\infty}\left(\frac{1}{z-a_{r}+m \omega}-\frac{1}{m}\right)\right\} .
\end{aligned}
$$

Therefore $\sum_{s=1}^{\infty} \Phi_{s}(z)$ has its sole poles at the points negatively congruent to the $\alpha$ 's (these points included), and each pole congruent to $\alpha_{r}$ is an infinity of exactly the same nature as the infinity $\alpha_{r}$ of $-\mu(z)$.

Hence, if $\mu(z)=\mu_{\alpha}(z)+\mu_{\beta}(z)$, the two meromorphic functions possessing respectively the sequences of poles negative and positive with respect to $\omega$, we see that

$$
-\left\{\mu_{a}(z)+g_{0}(z)\right\}-\left\{\mu_{\alpha}(z+\omega)+g_{1}(z)\right\}-\ldots-\left\{\mu_{a}(z+m \omega)+g_{m}(z)\right\}-\ldots,
$$

the functions $g(z)$ being integral functions of $z$, will have poles exactly like those of $\sum_{s=1}^{\infty} \Phi_{s}(z)$.

We can chose the functions $g(z)$ so that the series just written shall be convergent. For the poles of $\mu_{a}(z+m \omega)(m>k)$ will have moduli greater than $|k \omega|$, and hence, when $|z|<|k \omega|$, we shall have the expansion

$$
\mu_{a}(z+m \omega)=\sum_{s=0}^{\infty} \frac{z^{s}}{s !} \mu_{a}^{(s)}(m \omega) \text {. }
$$

If then we take

$$
M_{a}(z+m \omega)=-\mu_{a}(z+m \omega)+\sum_{s=0}^{s_{m}-1} \frac{z^{s}}{s !} \mu_{a}^{(s)}(m \omega),
$$

we shall have, when $|z|<|k \omega|$,

$$
M_{a}(z+m \omega)=-\sum_{s=s_{m}}^{\infty} \frac{z^{s}}{s !} \mu_{a}^{(s)}(m \omega) ;
$$

so that, by choosing $s_{m}$ sufficiently large, we may make $\left|M_{a}(z+m \omega)\right|<\epsilon_{m}$ where $\sum_{m=0}^{\infty} \epsilon_{m b}$ is absolutely convergent.

Then $\sum_{m=0}^{\infty} M_{a}(z+m \omega)$ is absolutely convergent except at the poles of $\sum_{i=1}^{\infty} \Phi_{s}(z)$, and only differs from the latter series of functions by an integral function.

SER. 2. VOL. 2. NO. 880 . 
Repesting the s8me argument for the $\beta$ 's and constructing the series of analogous functions

$$
M_{\beta}\{z-(m+1) \omega\}=\mu_{\beta}\{z-(m+1) \omega\}-\sum_{s=0}^{t_{m}-1} \frac{z^{s}}{s !} \mu_{\beta}^{(s)}\{-(m+1) \omega\},
$$

we see that

$$
\sum_{m=0}^{\infty}\left[M_{\mathrm{a}}(z+m \omega)+M_{\beta}\{z-(m+1) \omega\}\right]+G(z)
$$

is a solution of

$$
f(z+\omega)-f(z)=\mu(z) .
$$

It is a solution analogous to this which Hurwitz* has given.

22. We may now express the solution in a third form. We take, as before,

$$
\begin{aligned}
\mu(z)= & \sum_{r=1}^{\infty}\left[\sum_{s=1}^{k_{r}} \frac{a_{s}}{\left(z-a_{r}\right)^{3}}-\sum_{m=0}^{m_{r}-1}{ }_{r} c_{m} z^{m}\right] \\
& +\sum_{r=1}^{\infty}\left[\sum_{s=1}^{l_{r}} \frac{b_{s}}{\left(z-\beta_{r}\right)^{s}}-\sum_{m=0}^{n_{r}-1}{ }_{r} d_{m} z^{m}\right]+G(z),
\end{aligned}
$$

the a poles being negative and the $\beta$ poles positive with regard to $\omega$. And we construct the function

$$
\begin{array}{r}
Q(z)=-\sum_{m=0}^{\infty} \sum_{r=1}^{\infty} \sum_{s=1}^{k_{r}} a_{s}\left\{\frac{1}{\left(z-\alpha_{r}+m \omega\right)^{2}}+(-)^{s-1} \sum_{t=0}^{\sigma_{r}-1}\left(\begin{array}{c}
s+t-1 \\
t
\end{array}\right) \frac{z^{t}}{\left(\alpha_{r}-m \omega\right)^{s+t}}\right\} \\
+\sum_{m=1}^{\infty} \sum_{r=1}^{\infty} \sum_{s=1}^{l_{r}} b_{s}\left\{\frac{1}{\left(z-\beta_{r}-m \omega\right)^{s}}+(-)^{s-1} \sum_{t=0}^{s_{r}-1}\left(\begin{array}{c}
s+t-1 \\
t
\end{array}\right)\right. \\
\left.\times \frac{z^{t}}{\left(\beta_{r}+m \omega\right)^{s+t}}\right\}
\end{array}
$$

Consider the first series. If those values of $m$ and $r$ be omitted for which $|z| \leqslant\left|a_{r}-m \omega\right|$, the series converges with the group of series

$$
\sum_{m}^{\infty} \sum_{r}^{\infty} \sum_{t=s}^{\infty}\left(\begin{array}{c}
s+t-1 \\
t
\end{array}\right) \frac{z^{t}}{\left(a_{r}-m \omega\right)^{s+\ell}} \quad\left(s=1,2 \ldots, k_{r}\right) .
$$

Now, when $r$ is very large, $\left(\begin{array}{c}s+r-1 \\ r\end{array}\right)$ behaves to a first approximation like $\frac{1}{\Gamma(s)} r^{s-1}$ when $s>1$. Hence, by the same method of proof as that employed in $\$ 16$, the series are absolutely convergent provided ${ }_{s} \sigma_{r}>2 \rho_{r}$ for all values of $s$ and $r$, where $\rho_{r}$ is the integer next greater than the

\footnotetext{
* Hurwitz, Actı Mathematica, T. xx., pp. 308-311.
} 
order-number of the sequence $a_{1}, a_{2}, \ldots, a_{r}, \ldots$. When the order of the sequence is finite, so that $\rho_{r}=\rho$, we may take ${ }_{s} \sigma_{r}$ to be a finite quantity $\sigma$ where

$$
\sigma>2 \rho \text { and } \sigma>1 \text {. }
$$

Similar remarks apply to the second series.

The function $Q(z)$ is a meromorphic function, and, by suitable choice of the integral function $G(z)$, we may make $Q(z)+G(z)$ a solution of the difference equation

$$
f(z+\omega)-f(z)=\mu(z) .
$$

In each case the particular solution which we have obtained of this equation is a meromorphic function whose poles are two series of points, those negatively congruent to the poles of $\mu(z)$ negative with regard to $\omega$ (these poles being included), and those positively congruent to the poles of $\mu(z)$ positive with regard to $\omega$ (these points being excluded).

23. We have now completed the solution of the two fundamental subsidiary equations (A) and (B) of $\S 2$. Let us then consider the nature of the solution of

$$
\phi(z) f(z+\omega)-\chi(z) f(z)=\psi(z) .
$$

We have

$$
f(z)=\frac{f_{2}(z)}{f_{1}(z)}
$$

where

$$
f_{1}(z+\omega)-\frac{\phi(z)}{\chi^{(z)}} f_{1}(z)=0
$$

and

$$
f_{2}(z+\omega)-f_{2}(z)=\frac{\psi(z)}{\chi(z)} f_{1}(z) .
$$

Let the $n$-th positive and negative (with respect to $\omega$ ) zeros of $\phi(z)$, $\chi(z)$, and $\psi(z)$ respectively be $\alpha_{n}$ and $\alpha_{n}^{\prime}, \beta_{n}$ and $\beta_{n}^{\prime}, \gamma_{n}$ and $\gamma_{n}^{\prime}$. We have seen that we may take $f_{1}(z)$ to be a meromorphic function with poles at the sequences

$$
a_{n}^{\prime}-m \omega, \quad \beta_{n}+(m+1) \omega \quad(m=0,1, \ldots, \infty)
$$

and zeros at the sequences

$$
\alpha_{n}+(m+1) \omega, \quad \beta_{n}^{\prime}-m \omega,
$$

these poles and zeros being of the same order as the corresponding zeros of $\phi(z)$ and $\chi(z)$.

Hence $\frac{\psi(z)}{\chi(z)} f_{1}(z)$ has poles at the sequences

$$
\alpha_{n}^{\prime}-m \omega, \quad \beta_{n}+m \omega \quad(m=0,1, \ldots, \infty),
$$

these poles being of the same order as the corresponding zeros of $\phi(z)$ and $\chi(z)$. 
Consider now the solution of

$$
f_{2}(z+\omega)-f_{2}(z)=\frac{\psi(z)}{\chi(z)} f_{1}(z) .
$$

A particular solution will be a meromorphic function with its sole poles at the sequences

$$
a_{n}^{\prime}-n \omega, \quad \beta_{n}+(m+1) \omega \quad(m=0,1, \ldots, \infty) .
$$

These poles will be of the order of the corresponding zeros of $\phi(z)$ and $\chi(z)$, but not of the same type, i.e., the coefficients in the expansion near a pole will in general be different.

Finally, the particular solution $f(z)=\frac{f_{2}(z)}{f_{1}(z)}$ of the difference equation $\phi(z) f(z+\omega)-\chi(z) f(z)=\psi(z)$ will be a one-valued meromorphic function whose sole poles are at the sequences

$$
\beta_{n}^{\prime}-m \omega \quad(m=0,1, \ldots, \infty),
$$

each of the order of the corresponding zero $\beta_{n}^{\prime}$ of $\chi(z)$;

$$
a_{n}+(m+1) \omega \quad(m=0,1, \ldots, \infty),
$$

each of the order of the corresponding zero $a_{n}$ of $\phi(z)$.

The general solution of the difference equation

$$
\text { is } \quad \begin{gathered}
\phi(z) f(z+\omega)-\chi(z) f(z)=\psi(z) \\
f(z)+\varpi(z, \omega) / f_{1}(z)
\end{gathered}
$$

where $\varpi(z, \omega)$ is a simply periodic function of $z$ of period $\omega$.

It may be noticed that, unless $\varpi(z, \omega)$ has finite poles, this solution has its only poles at the sequences

$$
\beta_{n}^{\prime}-m \omega, \quad a_{n}+(m+1) \omega .
$$

It must be distinctly understood that we have given the nature of the general solution on the assumption that $\phi(z), \chi(z)$, and $\psi(z)$ are arbitrary integral functions of $z$. And the solution given may admit of reductions wbich will simplify its character when there are relations between the zeros of these functions.

24. As a single example of the general theory we may mention Prym's * solution of

$$
f(z+\omega)-z f(z)=c,
$$

which is

$$
f(z)=c e^{1 / \omega} \sum_{n=0}^{\infty} \frac{(-)^{n-1}}{n ! \omega^{n}(z+n \omega)} .
$$

Here $\alpha_{n}, a_{n}^{\prime}, \beta_{n}$ are non-existent, and $\beta_{1}^{\prime}=0$. 
1904.] The Linear DifFerence equation of the First order. 469

Other examples are furnished by the $G$-function," the multiple gamma functions, and the functions constructed by Mellin. ${ }^{+}$

As an example of the very different form which may be sometimes given to the general solution we may mention that, if $\phi(z), \chi(z)$, and $\psi(z)$ are all simply periodic functions of $z$ of period $\omega$, the solution of

$$
\phi(z) f(z+\omega)-\chi(z) f(z)=\psi(z)
$$

may be written $\frac{\psi(z)}{\phi(z)-\chi(z)}+\sigma(z, \omega)\left[\frac{\chi(z)}{\phi(z)}\right]^{z, \omega}$.

- See a paper by the author, Quarterly Journal of Mathematics, Vod. xxxI., pp. 264-314.

+ Mellin, scta Mathematica, T. xv., pp. 317-384. 